\title{
Der Einfluss experimentell variierter Zooplanktondichte auf die Produktion und Sedimentation im hocheutrophen See
}

\author{
Von H.R. Bürgi, H. Bührer, J. Bloesch und E. Szabo \\ Eidg. Anstalt für Wasserversorgung, Abwasserreinigung und Gewässerschutz (EAWAG), \\ CH-8600 Dübendorf
}

Manuskript eingegangen am 26. Februar 1979

\begin{abstract}
The infuence of experimentally varied zooplankton density on production and sedimentation in a highly eutrophic lake

Size-selective enrichment or exclusion of planktonic crustaceans significantly alters the succession of phytoplankton and smaller zooplankton. The cell density of algae (particularly of smaller forms) and the rate of production (life expectancy) of zooplankton increase sharply in the test units poor in zooplankton. In this primary production phase, the phosphate content of the water decreases within 10 days to $5 \%$ of its original value. Large herbivorous populations reduce the sedimentation rate of the organic substance, while increasing the $P: C$ ratio of the sediment.
\end{abstract}

\section{INHALTSVERZEICHNIS}

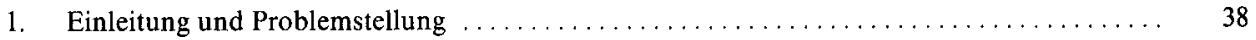

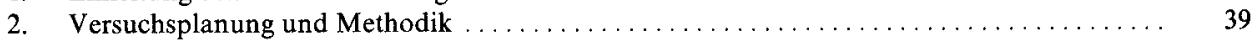

3. Resultate $\ldots \ldots \ldots \ldots \ldots \ldots \ldots \ldots \ldots \ldots \ldots \ldots \ldots \ldots \ldots \ldots \ldots \ldots \ldots \ldots \ldots \ldots \ldots \ldots \ldots \ldots$

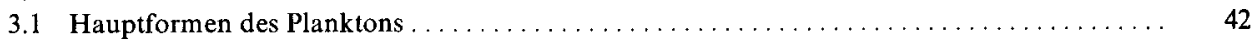

3.2 Zeitreihen der Algen, Protozoen, Rotatorien und Crustaceen $\ldots \ldots \ldots \ldots \ldots$

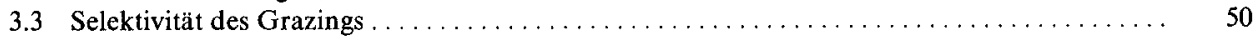

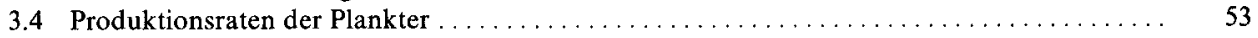

3.5 Freisetzung und Sedimentation der Nährstoffe $\ldots \ldots \ldots \ldots \ldots \ldots \ldots \ldots \ldots \ldots \ldots \ldots \ldots \ldots$

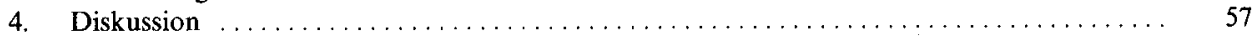

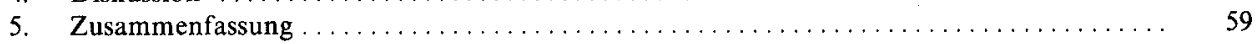

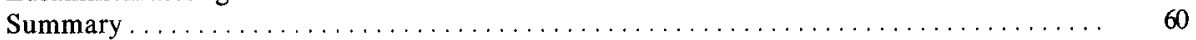

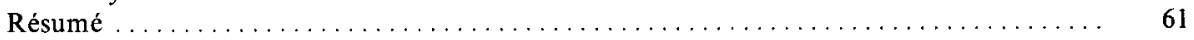

Literaturverzeichnis $\ldots \ldots \ldots \ldots \ldots \ldots \ldots \ldots \ldots \ldots \ldots \ldots \ldots \ldots \ldots \ldots \ldots \ldots$

\section{Einleitung und Problemstellung}

Die Zusammenhänge zwischen der Nährstoffbelastung eines Sees und seiner Biomasseproduktion spielen bei der Beurteilung seines trophischen Zustands eine wichtige Rolle. Die Zufuhr der Nährstoffe aus allochthonen Quellen ist nur ein einziger Faktor neben vielen andern (Licht, Temperatur, Turbulenz, Nährstoffrezirkulation), welche die Produktion organischer Substanz steuern. Mit empirischen 
Ansätzen allgemeingültige Beziehungen zwischen Trophiegrad und Nährstoffbelastung eines Gewässers $\mathrm{zu}$ beschreiben [43, 44], gelingt nur, wenn ein statistisch genügendes Material aus vielen Seen vorhanden ist. Die Anwendung dieser allgemeinen Ansätze auf einen einzelnen See versagt nun aber oft, weil dessen Morphologie, aber auch klimatische Faktoren, ungenügend berücksichtigt bleiben. Zur Erklärung temporärer Phänomene (z. B. Algenblüten) können diese Beziehungen überhaupt nicht verwendet werden.

Die netzartige Verknüpfung der Zustandsgrössen und Wirkungsfaktoren im limnischen Ökosystem lässt sich nur durch ein integrales Modell nachvollziehen. Wird der See an einer einzigen Stelle beeinflusst, reagiert das System als Ganzes. Mit numerischen Modellen lassen sich Austauschvorgänge, Rezirkulation der Nährstoffe und Primärproduktion zwar gut beschreiben. Die biologische Reaktion des mit den Algen eng verknüpften Zooplanktons bzw. dessen Rückwirkungen auf die Primärproduktion werden jedoch weitgehend vernachlässigt [11, 19]. Die gegenseitigen Abhängigkeiten des Phytoplanktons und des Zooplanktons wurden zwar schon früh erkannt und mehrfach beschrieben $[2,8,21,23,28,36,42]$, es sind aber nur wenige quantitative Analysen, und auch diese nur von Teilaspekten der Wechselwirkungen, erarbeitet worden [16, 17, 20, 27, 29-31, 33, 34]. Die vorliegenden Versuche hatten zum Ziel, die Rolle des Zooplanktons im limnischen Stoffkreislauf zu quantifizieren. Die folgenden Teilfragen sollten vertieft studiert werden:

- Wie stark verändert das Zooplankton durch Abweiden (Grazing) die Algenbiozönose?

- Hat die Variation der Zooplanktondichte Auswirkungen auf die chemische Zusammensetzung (Stöchiometrie) der Algen und des Sediments?

- Wie stark beeinflusst das Zooplankton das Nährstoffangebot im Epilimnion?

- Welche direkten und indirekten Wechselwirkungen spielen zwischen den verschiedenen Zooplanktonkompartimenten (Protozoen, Rotatorien, herbivoren Crustaceen und karnivoren Crustaceen)?

\section{Versuchsplanung und Methodik}

Um die Extrapolation der Resultate auf das Geschehen im See zu ermöglichen, galt es, unter naturnahen Bedingungen zu arbeiten. $\mathrm{Zu}$ diesem $\mathrm{Zwecke}$ wurde eine Insitu-Versuchseinrichtung mit 25001 Probenvolumen benutzt (Abb. 1), vergleichbar den Plastikcontainern von Goldman [12], Strickland [38] und Bürgi [6]. Einige Kultureinheiten wurden unten mit Planktonnetzen (Maschenweite $95 \mu \mathrm{m}$ und $200 \mu \mathrm{m})$ bespannt. Damit wurde das Plankton des Sees beim Einhängen der

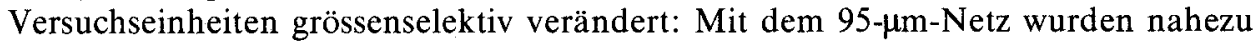
alle Crustaceen ausfiltriert; einzig wenige kleinste Jugendstadien der Crustaceen verblieben neben Protozoen und Rotatorien in diesen Containern. Durch die 200- $\mu$ Netze schlüpften grössere Jugendstadien der Kleinkrebse durch. Erwachsene Tiere fanden sich aber praktisch noch keine in diesen Kultureinheiten.

In den Kontrolleinheiten befand sich die unveränderte Zusammensetzung des Seenplanktons, und in den angereicherten Einheiten waren adulte Tiere - mit grobmaschigen Netzen im umgebenden Wasser gesammelt - angereichert. 


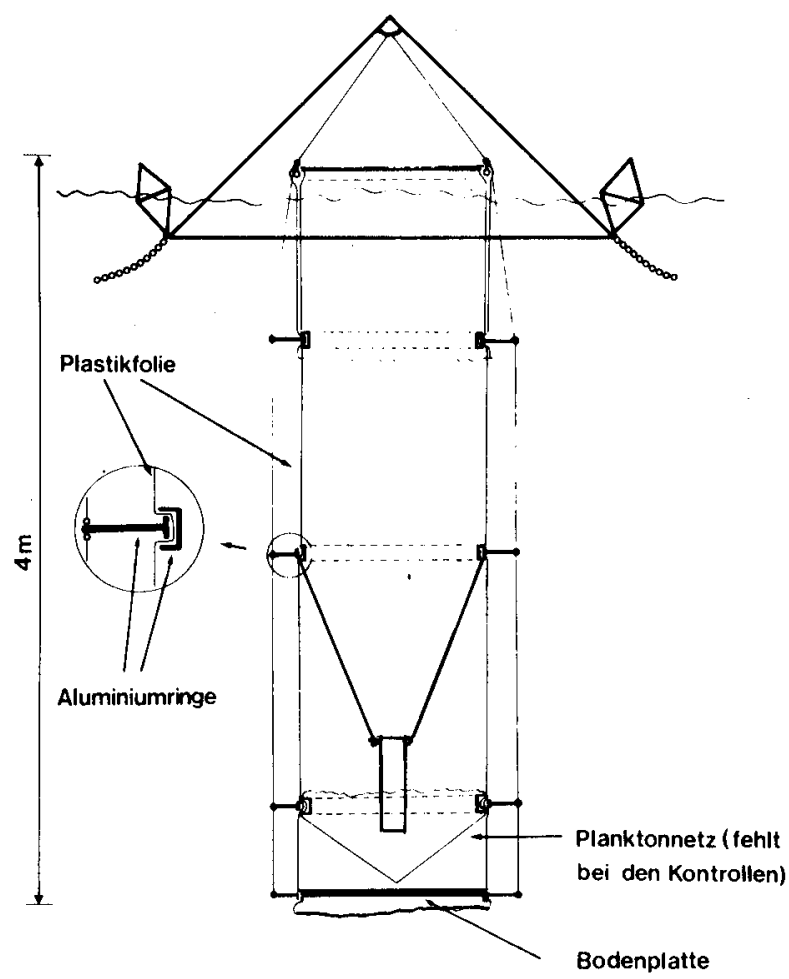

Abb. 1. Schema der 25001 fassenden Versuchseinrichtung in situ.

Figure 1. Culture unit in situ.

Das Phytoplankton wurde durch diese Eingriffe nicht verändert. Der hocheutrophe Greifensee enthielt zur Versuchszeit praktisch nur kleine Algen $(<50 \mu \mathrm{m}$ Durchmesser), welche bei den verwendeten Maschenweiten die Netze passierten. Alle Versuchseinheiten wurden nach der Exposition mit Sedimentauffanggefässen ausgerüstet und mit Bodenplatten geschlossen.

Das Versuchsgewässer (Greifensee) enthielt zur Versuchszeit genügend Phosphor, um eine Nährstofflimitierung auszuschliessen. Insgesamt wurden sieben Versuche mit der oben beschriebenen Kulturmethode durchgeführt. In der vorliegenden Arbeit werden drei davon beschrieben. Die Resultate der übrigen Versuche stimmen im Verlauf weitgehend mit einem dieser Versuche überein.

\section{Versuchsplan}

Versuch I (3.-17. September 1974)

3 Parallelen mit 95- $\mu \mathrm{m}-\mathrm{Netz}$

3 Parallelen mit $200-\mu \mathrm{m}-\mathrm{Netz}$

3 Parallelen ohne Netz als Vergleichseinheiten (Kontrollen) 
Versuch II (29. Mai bis 12. Juni 1975)

1 Einheit mit $95-\mu \mathrm{m}-\mathrm{Netz}$

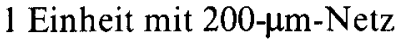

1 Kontrolle unverändert

2 Einheiten mit Zooplankton angereichert

Versuch III (14.-29. Juni 1976)

Wie Versuch II

Der erste Versuch diente der Ermittlung der (noch unbekannten) Streuung der Methode. Die deutlichen Unterschiede, die sich zwischen filtrierten Einheiten und Kontrollen bzw. angereicherten Versuchseinheiten einsteliten (vgl. Abb.2), zeigten, dass künftig auf eine streng statistische Versuchsplanung verzichtet werden konnte.

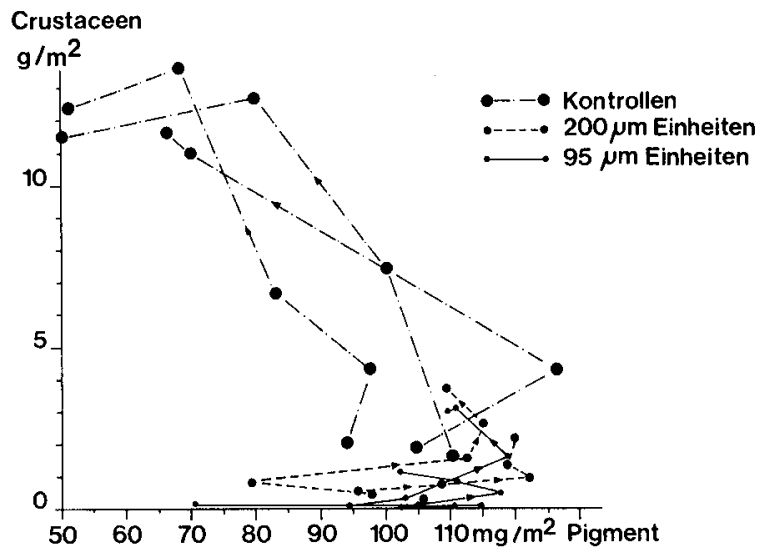

Abb.2. Phasendiagramme Versuch I: Algen versus Crustaceen. Die Pfeile markieren die zeitliche Abfolge. Zwischen zwei Punkten liegen 3-4 Tage.

Figure 2. Experiment I. Phase diagrams: Algae versus crustaceans. The arrows indicate the time sequence. $3-4$ days lie between two points.

Das grosse Probenvolumen der Versuchseinheiten gestattete das Arbeiten mit üblichen limnologischen Geräten. Die Proben wurden aus 0, 1, 2 und $3 \mathrm{~m}$ Tiefe geschöpft. Für einzelne Analysen wurden die Einzelproben zu einer gewichteten Mischprobe vereinigt. Das Zooplankton wurde mit einem Spezialnetz $\left(50 \mathrm{~cm}^{2}\right.$ Eintrittsöffnung, $1700 \mathrm{~cm}^{2}$ Filterfläche, $95 \mu \mathrm{m}$ Maschenweite) in je acht Netzzügen vom Boden bis zur Oberfläche geschöpft. Um Schwarmbildungen der Zooplankter zu kompensieren, wurden die acht Züge regelmässig auf den Querschnitt der Kultureinheiten verteilt.

Analysenmethoden

Phytoplankton: Zählung im umgekehrten Mikroskop nach Utermöhl [41]

Zooplankton: Zählung unter der Binokularlupe (Crustaceen) bzw. wie Phytoplankton (Rotatorien, Protozoen) 
Pigmente: Nach Goltermann [13]

Phosphat-P: Nach Ambühl und Schmid [1]

Gesamt-P: Nach Schmid und Ambühl [35]

Sediment: Gravimetrische Messung der Anteile vor und nach Glühen

Sichttiefe: Secchi-Scheibe

Strahlung unter Wasser: Mit dem Quantum-Sensor Lambda (nur 400-700 nm)

\section{Resultate}

\subsection{Hauptformen des Planktons}

Die Artenlisten (Tab. 1 und 2) enthalten nur die Arten mit hohen Individuendichten oder grosser Biomasse.

Tabelle 1. Beobachtete und gezählte Phytoplanktonformen und ihr Umrechnungsvolumen für Nassgewichtbiomasse.

Table 1. Observed and counted phytoplankton and their conversion factors (as volume) for determination of the wet-weight biomass.

\begin{tabular}{|c|c|c|c|c|}
\hline \multirow[t]{2}{*}{ Gattung, Art } & \multicolumn{3}{|c|}{ Beobachtet in Versuch } & \multirow[t]{2}{*}{ Volumen/Zelle in $\mu \mathrm{m}^{3}$} \\
\hline & I & II & III & \\
\hline \multicolumn{5}{|l|}{ Cyanophyceae } \\
\hline Anabaena flos-aquae & $\mathrm{x}$ & & $\mathrm{x}$ & 190 \\
\hline \multicolumn{5}{|l|}{ Aphanizomenon flos- } \\
\hline aquae fo. gracile Elenk & $\mathrm{x}$ & & $\mathrm{x}$ & 90 \\
\hline \multicolumn{5}{|l|}{ Dinophyceae } \\
\hline Ceratium hirundinella & $\mathrm{x}$ & $\mathrm{x}$ & $\mathrm{x}$ & 45000 \\
\hline \multicolumn{5}{|l|}{ Peridinium cinctum } \\
\hline fo. angulatum & $\mathrm{x}$ & $\mathrm{x}$ & $\mathrm{x}$ & 25000 \\
\hline \multicolumn{5}{|l|}{ Chlorophyceae } \\
\hline \multicolumn{5}{|l|}{ Ankyra ancora (G.M. Smith) } \\
\hline Fott fo. spinosa & $\mathrm{x}$ & $\mathrm{x}$ & $\mathrm{x}$ & 200 \\
\hline Coelastrum microporum & $\mathrm{x}$ & $\mathrm{x}$ & $\mathrm{x}$ & 250 \\
\hline \multicolumn{5}{|l|}{ Coelastrum cambricum } \\
\hline var. intermedium West & $\mathrm{x}$ & & $\mathrm{x}$ & 300 \\
\hline Oocystis lacustris & $\mathrm{x}$ & $\mathrm{x}$ & $\mathrm{x}$ & 200 \\
\hline Dictyosphaerium pulchellum & $\mathrm{x}$ & $\mathrm{x}$ & $\mathrm{x}$ & 250 \\
\hline Elakatothrix gelatinosa & $\mathrm{x}$ & $\mathrm{x}$ & $\mathrm{x}$ & 80 \\
\hline \multicolumn{5}{|l|}{ Pediastrum duplex } \\
\hline Pediastrum boryanum & $\mathrm{x}$ & $\mathrm{x}$ & $\mathrm{x}$ & 300 \\
\hline \multicolumn{4}{|l|}{ Botryococcus braunii } & \\
\hline Phacotus lendneri & $\mathrm{x}$ & & & 150 \\
\hline \multicolumn{5}{|l|}{ Planktosphaeria gelatinosa } \\
\hline Pseudosphaerocystis lacustris & $\mathrm{x}$ & & & 200 \\
\hline \multicolumn{5}{|l|}{ Tetrachlorella alternans } \\
\hline \multicolumn{5}{|l|}{ Pandorina morum } \\
\hline \multicolumn{5}{|l|}{ Conjugatophyceae } \\
\hline Cosmarium subprotumidum & & & & \\
\hline var. gregori W. et W. & $\mathrm{x}$ & $\mathrm{x}$ & & 7500 \\
\hline
\end{tabular}




\begin{tabular}{|c|c|c|c|c|}
\hline \multirow[t]{2}{*}{ Gattung, Art } & \multicolumn{3}{|c|}{ Beobachtet in Versuch } & \multirow[t]{2}{*}{ Volumen/Zelle in $\mu \mathrm{m}^{3}$} \\
\hline & $\mathrm{I}$ & II & III & \\
\hline \multicolumn{5}{|l|}{ Cosmarium subcostatum } \\
\hline fo. minor W. et W. & $\mathrm{x}$ & $\mathrm{x}$ & & 7500 \\
\hline Cosmarium depressum & & & & \\
\hline var. planctonicum Reverd. & & & $\mathrm{x}$ & 2500 \\
\hline \multicolumn{5}{|l|}{ Closterium acutum } \\
\hline var. variabile & $\mathrm{x}$ & $\mathrm{x}$ & & 800 \\
\hline Closterium sp. & & & $\mathrm{x}$ & 4500 \\
\hline Staurastrum sebaldi ornatum & $\mathrm{x}$ & & & 11000 \\
\hline Staurastrum cingulum var. obesum & $\mathrm{x}$ & & & 1100 \\
\hline \multicolumn{5}{|l|}{ Cryptophyceae } \\
\hline Cryptomonas erosa & $x$ & $\mathbf{x}$ & $\mathrm{x}$ & 2100 \\
\hline Cryptomonas ovata & $\mathrm{x}$ & $\mathrm{x}$ & $\mathrm{x}$ & 3500 \\
\hline Rhodomonas lacustris & $x$ & $\mathrm{x}$ & $\mathrm{x}$ & 200 \\
\hline Katablepharis ovalis & $\mathrm{x}$ & & & 250 \\
\hline \multicolumn{5}{|l|}{ Diatomeae } \\
\hline Asterionella formosa & $\mathrm{x}$ & & & 400 \\
\hline Cyclotella bodanica & & & $\mathrm{x}$ & 5000 \\
\hline Cyclotella sp., klein & & $\mathrm{x}$ & & 100 \\
\hline Nitzschia acicularis & & $\mathrm{x}$ & & 200 \\
\hline
\end{tabular}

Tabelle 2. Beobachtete und gezählte Zooplanktonformen und ihr Umrechnungsvolumen für Nassgewichtbiomasse.

Table 2. Observed and counted zooplankton and their conversion factors (as volume) for determination of the wet-weight biomass.

\begin{tabular}{|c|c|c|c|c|}
\hline \multirow[t]{2}{*}{ Gattung, Art } & \multicolumn{3}{|c|}{ Beobachtet in Versuch } & \multirow[t]{2}{*}{ Ind.-Volumen in $10^{6} \mu \mathrm{m}^{3}$} \\
\hline & $\mathrm{I}$ & II & III & \\
\hline \multicolumn{5}{|l|}{ Protozoa } \\
\hline Coleps hirtus & $\mathrm{x}$ & & & 0,02 \\
\hline Strobilidium gyrans & $\mathrm{x}$ & $\mathrm{x}$ & $\mathrm{x}$ & 0,015 \\
\hline Askenasia volvox & & & $\mathrm{x}$ & 0,025 \\
\hline Strombidinopsis sp. & & $\mathrm{x}$ & $\mathrm{x}$ & 0,02 \\
\hline Strombidium viride & & $\mathrm{x}$ & $\mathrm{x}$ & 0,03 \\
\hline \multicolumn{5}{|l|}{ Rotatoria } \\
\hline Gruppe Keratella cochlearis & $\mathrm{x}$ & $\mathrm{x}$ & $\mathrm{x}$ & 0,035 \\
\hline Gruppe Keratella quadrata & $\mathrm{x}$ & $\mathrm{x}$ & $\mathrm{x}$ & 0,1 \\
\hline Polyarthra sp. & & $\mathbf{x}$ & $\mathrm{x}$ & 0,45 \\
\hline Asplanchna priodonta & & $\mathrm{x}$ & $\mathrm{x}$ & 40 \\
\hline \multicolumn{5}{|l|}{ Phyllopoda } \\
\hline \multicolumn{5}{|c|}{ Gruppe Daphnia hyalina-galeata } \\
\hline Jugendstadien, klein & $\mathrm{x}$ & $\mathrm{x}$ & $\mathrm{x}$ & 15 \\
\hline Jugendstadien, mittel & & & & 21 \\
\hline Jugendstadien, gross & & & & 23 \\
\hline Adult & & & & 90 \\
\hline Daphnia cucullata (mittel) & & $\mathrm{x}$ & & 20 \\
\hline
\end{tabular}




\begin{tabular}{llccc}
\hline Gattung, Art & \multicolumn{2}{l}{ Beobachtet in Versuch } & & Ind.-Volumen in $1^{6} \mu \mathrm{m}^{3}$ \\
& $\mathrm{I}$ & II & III & \\
\hline $\begin{array}{l}\text { Copepoda } \\
\text { Eudiaptomus gracilis }\end{array}$ & & & & 40 \\
juvenil $\left(\mathrm{C}_{1}-\mathrm{C}_{3}\right)$ & $\mathrm{x}$ & $\mathrm{x}$ & $\mathrm{x}$ & 200 \\
$\mathrm{C}_{4}$, adult & $\mathrm{x}$ & $\mathrm{x}$ & $\mathrm{x}$ & 45 \\
Cyclops strenuus, juvenil & $\mathrm{x}$ & & & 200 \\
Cyclops strenuus, adult & & $\mathrm{x}$ & $\mathrm{x}$ & 45 \\
Cyclops abyssorum, juvenil & $\mathrm{x}$ & & & 200 \\
Cyclops abyssorum, adult & $\mathrm{x}$ & $\mathrm{x}$ & $\mathrm{x}$ & 75 \\
Mesocyclops leuckarti, adult & $\mathrm{x}$ & & & 7,5 \\
Nauplien & $\mathrm{x}$ & $\mathrm{x}$ & $\mathrm{x}$ & \\
\hline
\end{tabular}

\subsection{Zeitreihen der Algen, Protozoen, Rotatorien und Crustaceen}

Die Entwicklungsgänge von Phytoplankton und Zooplankton weisen auf typische Räuber-Beute-Beziehungen zwischen verschiedenen Organismengruppen hin.

Das Ausfiltrieren (= Abwesenheit) der Crustaceen führt zu starkem Algenwachstum und - leicht phasenverschoben - zu einer Vermehrung der Protozoen und Rotatorien (Abb. 5a, b, 6a, b).

Das Algenmaximum wird meistens zwischen dem 6. und 9. Versuchstag erreicht, nur in einem Experiment hält die Zunahme bis zum Schluss des Experiments an.

Über die Versuchszeit gemittelt wurden die folgenden Pigmentgehalte (mg Chlorophyll $/ \mathrm{m}^{2}$ ) gemessen:

\begin{tabular}{llcc}
\hline & $95-\mu \mathrm{m}$-Einheiten & $200-\mu \mathrm{m}$-Einheiten & Kontrollen \\
\hline Versuch I & 29,3 & 31,2 & 23,8 \\
Versuch II & 112 & 114 & 64 \\
Versuch III & 244 & 168 & 54 \\
\hline
\end{tabular}

Mit dem Pigmentgehalt korrespondierend ging auch die Sichttiefe in den ausfiltrierten Einheiten rapide von 2 bis $3 \mathrm{~m}$ Ausgangswert auf $1 \mathrm{~m}$ und weniger zurück (Abb.5a, b, 6a, b).

Die Sichttiefe (reziprok) korreliert gut mit dem totalen Pigmentgehalt. Es besteht jedoch keine gesicherte Abhängigkeit von der Gesamtbiomasse.

Hohe Crustaceendichten unterdrücken Algen, Protozoen und Rotatorien gleichermassen (Abb.6c, d). In den angereicherten Testeinheiten unterliegt das Crustaceenplankton zunächst einer Sterbephase. Als Ursache für das Verschwinden der Crustaceen kommt neben Nahrungsmangel und mechanischer Schädigung (Netzfänge) auch die Predation in Betracht. Die Anreicherung der Zooplankter verringert die Distanz von räuberischen und herbivoren Crustaceen und begünstigt damit den Räuber. Nach Filmdokumenten von Strickler [39] vermögen Copepoden ihre Beute anhand feinster Schwingungen im Wasser auf Distanzen von mehreren Zentimetern zu orten.

Die Beziehungen zwischen Algen und Crustaceen können in einem Phasendiagramm veranschaulicht werden (Abb.2 und 3), das schon von Volterra und Lotka entwickelt wurde [45]. 


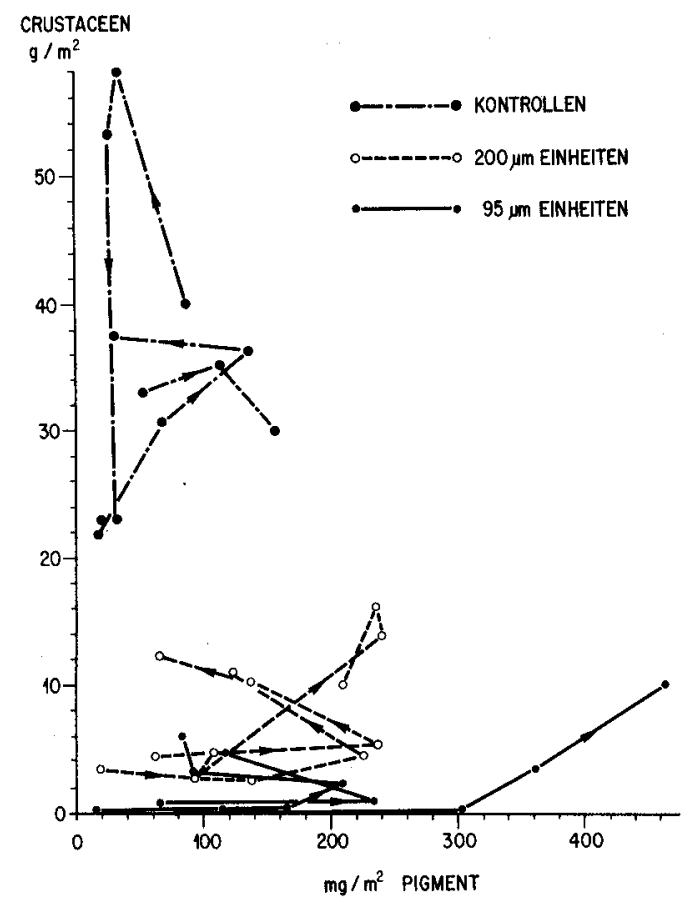

Abb.3. Phasendiagramme Versuche II und III: Algen versus Crustaceen. Die Pfeile markieren die zeitliche Abfolge. Zwischen zwei Punkten liegen 3-4 Tage.

Figure 3. Experiments II and III: Phase diagrams algae versus crustaceans. The arrows indicate the time sequence. 3-4 days lie between two points.

Geringe Zooplanktondichten erlauben den Algen, bei günstigen Nährstoff- und Lichtbedingungen (z. B. im Frühjahr) exponentiell zu wachsen. Auf die Verbesserung des Nahrungsangebots reagieren die herbivoren Zooplankter mit intensiver Vermehrung. Entsprechend der Trefferwahrscheinlichkeit zwischen Herbivoren und Algen nimmt der Frassverlust zu; schliesslich übersteigen die Verluste durch Frass, Sedimentation usw. die Primärproduktion, und die Algenbiomasse fällt zusammen. Das Zooplankton kann vorerst weiterwachsen, bis die Nahrungskonzentration im täglich filtrierten Wasser den Energiebedarf für den Stoffwechsel nicht mehr deckt. Nach Metz erneuern Daphnien einen Grossteil ihres C-Inhalts täglich [25]. Fällt das Nahrungsangebot auf etwa $1 \mathrm{~g} / \mathrm{m}^{3}$ Algennassgewicht $=\sim 3 \mathrm{mg} / \mathrm{m}^{3}$ Pigment, hungern viele herbivoren Tiere. Im Hungerzustand produzieren Daphnien Dauerstadien (Ephippien). Ephippienbildung konnte in allen Versuchseinheiten mit geringer Algendichte, aber auch im See zur Zeit des «Klarwasserstadiums», beobachtet werden.

Die geringste Dynamik zeigen Einheiten des ersten Versuchs. Hier erlaubte der geringe Nährstoffgehalt keine hohe Primärproduktion. Gegenüber den Kontrollen (mit deutlichem Grazing) ergeben sich aber auch hier signifikante Unterschiede. 
Klimatische Schwankungen tragen ihrerseits zur Dynamik der Algenentwicklung bei: Schlechtes Wetter bewirkte in der Startphase des Versuchs III in allen Testeinheiten eine Abnahme des Pigmentgehalts. Die hohen Phosphatkonzentrationen müssten unter besseren Lichtbedingungen in den Einheiten ohne Crustaceen früher zu einer Hochproduktion führen. Diese folgt dann der anfänglichen Depression und bringt innerhalb der nächsten 10 Tage eine Steigerung des Pigmentgehalts um das Zehnfache (Abb.6a). Hohe Crustaceenbestände vermögen aber umgekehrt in einer Schönwetterphase des zweiten Versuchs die Algenbiomasse nicht zu verringern. Die Abweideverluste konnten in diesem Fall durch hohe Primärproduktionsraten kompensiert werden.

Der Rückgang der Algenbiomasse gegen Versuchsende (Abb.5a, b) hängt nicht nur mit Grazing-Verlusten zusammen, sondern ist zu einem guten Teil durch die Sedimentation der Algen bedingt. Wegen dieser Sedimentation verarmt das Wasser innerhalb kurzer Zeit an Nährstoffen, und Phosphat wird zum wachstumsbegrenzenden Faktor. Innerhalb von 14 Tagen werden bis 97\% des Anfangsgehalts an $\mathrm{PO}_{4}-\mathrm{P}$ durch Algen gezehrt. Ein wesentlicher Teil davon sedimentiert (inkorporiert in Algen) ohne Rezirkulation.

Wieweit durch die Kulturmethode die Eddy-Diffusionskoeffizienten und die Sedimentationsgeschwindigkeit verändert werden, ist unbekannt. Die Temperatur und Stoffgradienten sind zwar in den Kultureinheiten in der gleichen Grössenordnung wie im See, doch fehlen scharfe Grenzen, wie sie im See beobachtet werden können (Einschichtungsphänomene, horizontale Strömungen, Seiches).

Aus Gründen der Übersichtlichkeit wurden die Crustaceen und Rotatorien in den Abb.4-6 nicht in herbivore und karnivore Tiere unterteilt. Das Verhältnis bleibt während der Versuchszeit nicht konstant. Mehrere Faktoren bewirken eine Verschiebung zugunsten der Karnivoren:

- In den filtrierten Einheiten wachsen die omnivoren Jugendstadien der Copepoden zu karnivoren Adulten aus. Die Versuchszeit reicht jedoch nicht für einen vollständigen Entwicklungszyklus aus, so dass eine Verjüngung der Population ausbleibt.

- In den angereicherten Kulturen (und zum Teil in den Kontrollen) wird die Fluchtdistanz der Herbivoren zu den Karnivoren als Resultat der Anreicherung bzw. hohen Sekundärproduktion verringert, was zu erhöhter Predation führt.

- Die herbivoren Tiere bekommen den Nahrungsmangel - die Folge übermässigen Grazings - früher zu spüren als die Räuber, deren Nahrungsangebot noch sehr hoch ist.

Protozoen und Rotatorien werden durch die Crustaceen in zweifacher Hinsicht beeinflusst: Direkt durch Predation, indirekt durch Nahrungskonkurrenz. Beide Selektionsmechanismen können festgestellt werden. Im Versuch III führt das Aufkommen der Crustaceen zu einem rapiden Zusammenbruch von Protozoen und Rotatorien, obwohl die Algenbiomasse hoch ist oder weiter zunimmt (Abb.6a, b). Im Versuch II wachsen dagegen selbst bei hohen Crustaceenbeständen viele Protozoen und Rotatorien, solange für sie genügend Nahrung (Nannoplankter) da ist (Abb. 5c, d). Der Grazing-Effekt der Protozoen und Rotatorien ist kaum messbar und wenn, dann nur bei den kleinsten Algen (vgl. Abb. 8b). 


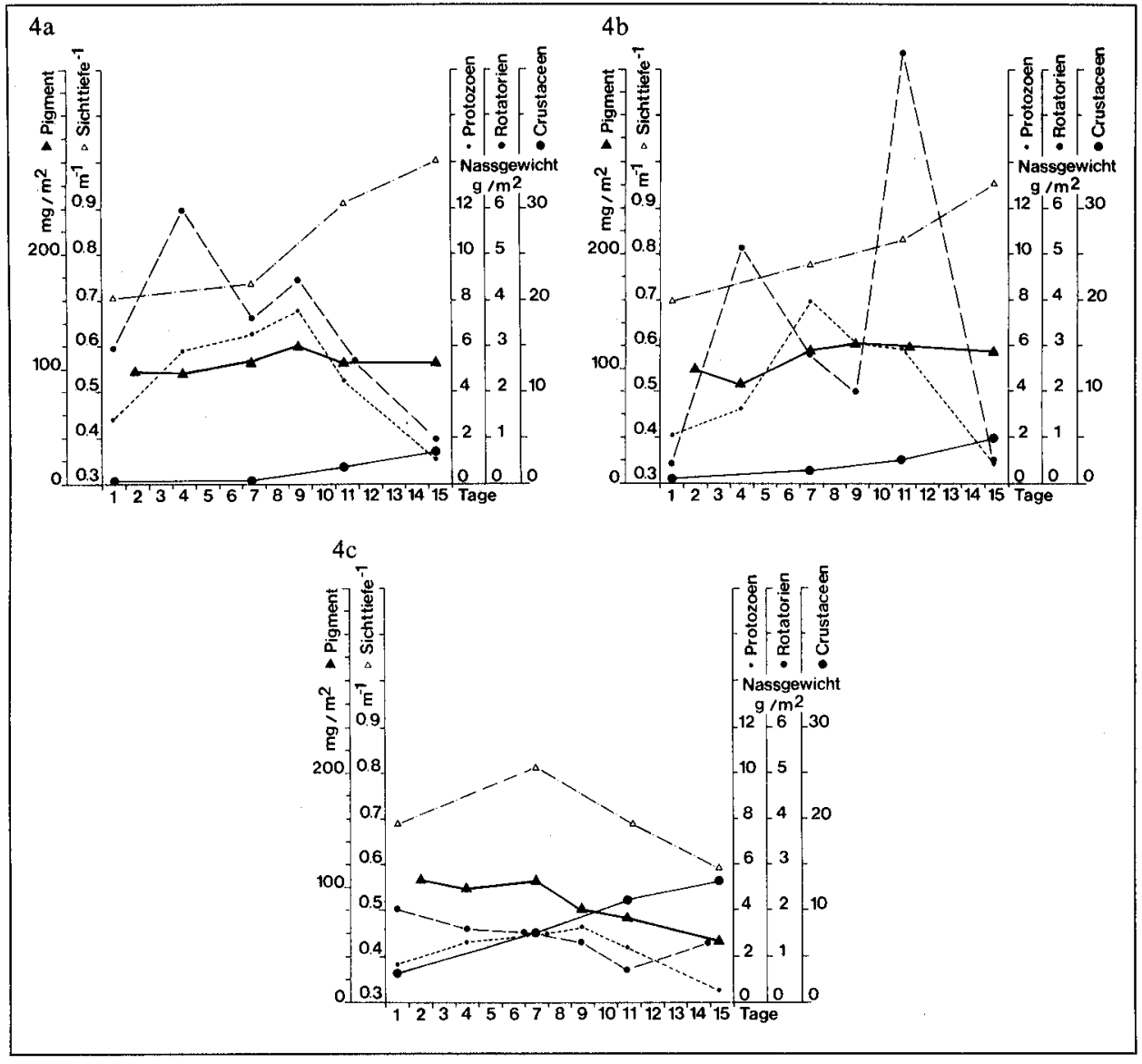

Abb.4. Versuch I: Zeitreihen der Planktongruppen.

Figure 4. Experiment I: Succession of different planktonic groups.

Abb. 4a. Crustaceenplankton, $>95 \mu \mathrm{m}$, ausfiltriert. Mittelwerte aus drei Parallelen.

Figure 4a. Planktonic crustaceans filtered out through a $95-\mu \mathrm{m}$ net. Mean of three parallels.

Abb. 4b. Crustaceenplankton, $>200 \mu \mathrm{m}$, ausfiltriert. Mittelwerte aus drei Parallelen.

Figure 4b. Planktonic crustaceen filtered out through a $200-\mu \mathrm{m}$ net. Mean of three parallels.

Abb.4c. Unveränderte Biozönose. Mittelwerte aus drei Parallelen.

Figure 4c. Unaltered biocenosis. Mean of three parallels.

Auch zwischen Protozoen und Rotatorien zeichnen sich Räuber-Beute-Beziehungen ab: Hohe Rotatorienbestände im Versuch III verhindern das Aufkommen der Protozoen (Abb.6a, 6b). Wieweit die einseitige Futterzusammensetzung (weitgehendes Fehlen der Ultraplankter) die Protozoen am Aufkommen hindert, ist nicht bekannt. 


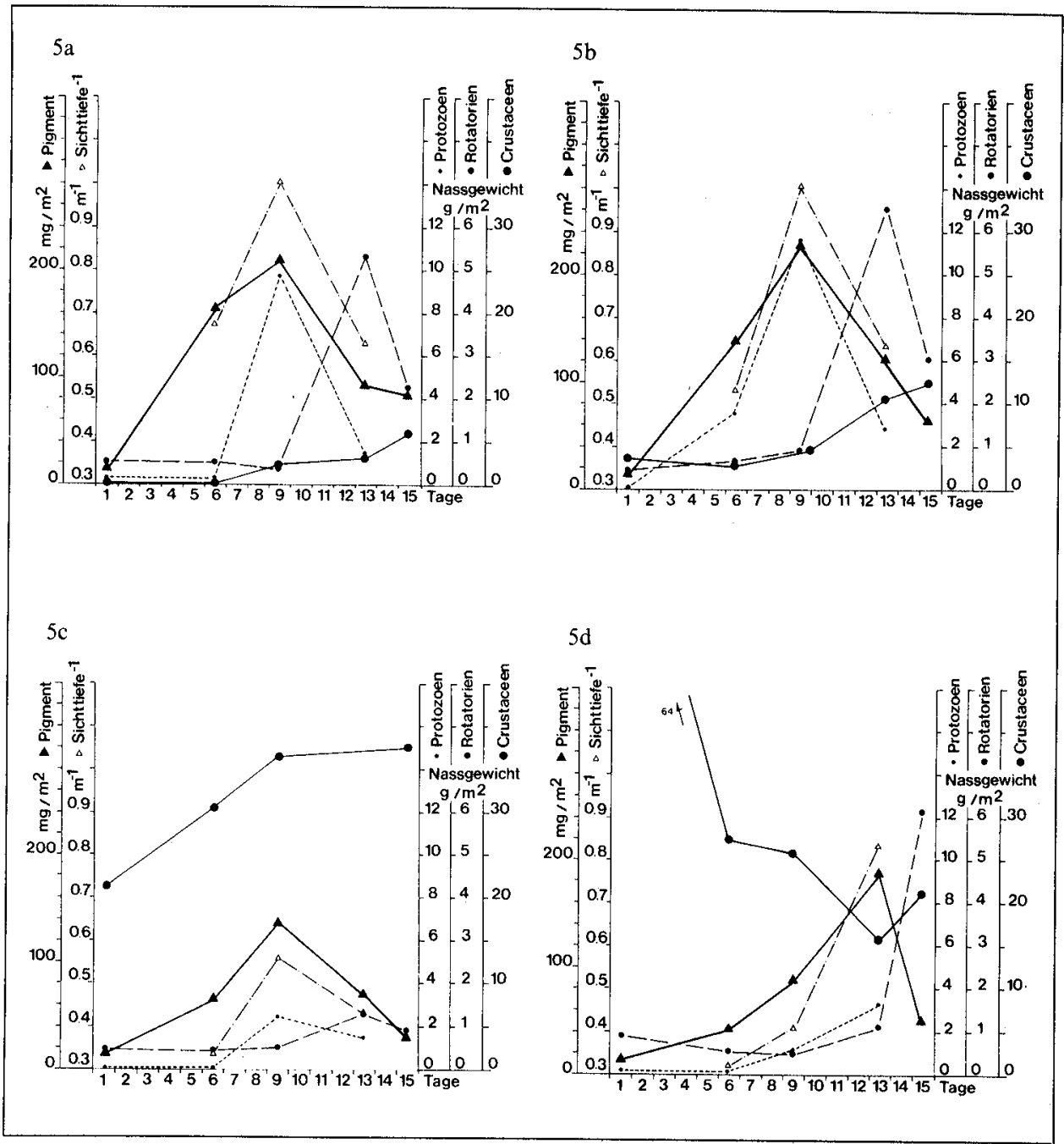

Abb.5. Versuch II: Zeitreihen der Planktongruppen.

Figure 5. Experiment II: Succession of different planktonic groups.

Abb. 5a. Crustaceenplankton, $>95 \mu \mathrm{m}$, ausfiltriert.

Figure 5a. Planktonic crustaceans filtered out through a $95-\mu \mathrm{m}$ net.

Abb. 5 b. Crustaceenplankton, $>200 \mu \mathrm{m}$, ausfiltriert.

Figure $5 \mathrm{~b}$. Planktonic crustaceans filtered out through a $200-\mu \mathrm{m}$ net.

Abb. 5 c. Biozönose, unverändert.

Figure $5 \mathrm{c}$. Unaltered biocenosis.

Abb.5d. Crustaceenplankton, angereichert. Mittelwert aus zwei Parallelen.

Figure 5d. Enriched with planktonic crustaceans. Mean of two parallels. 


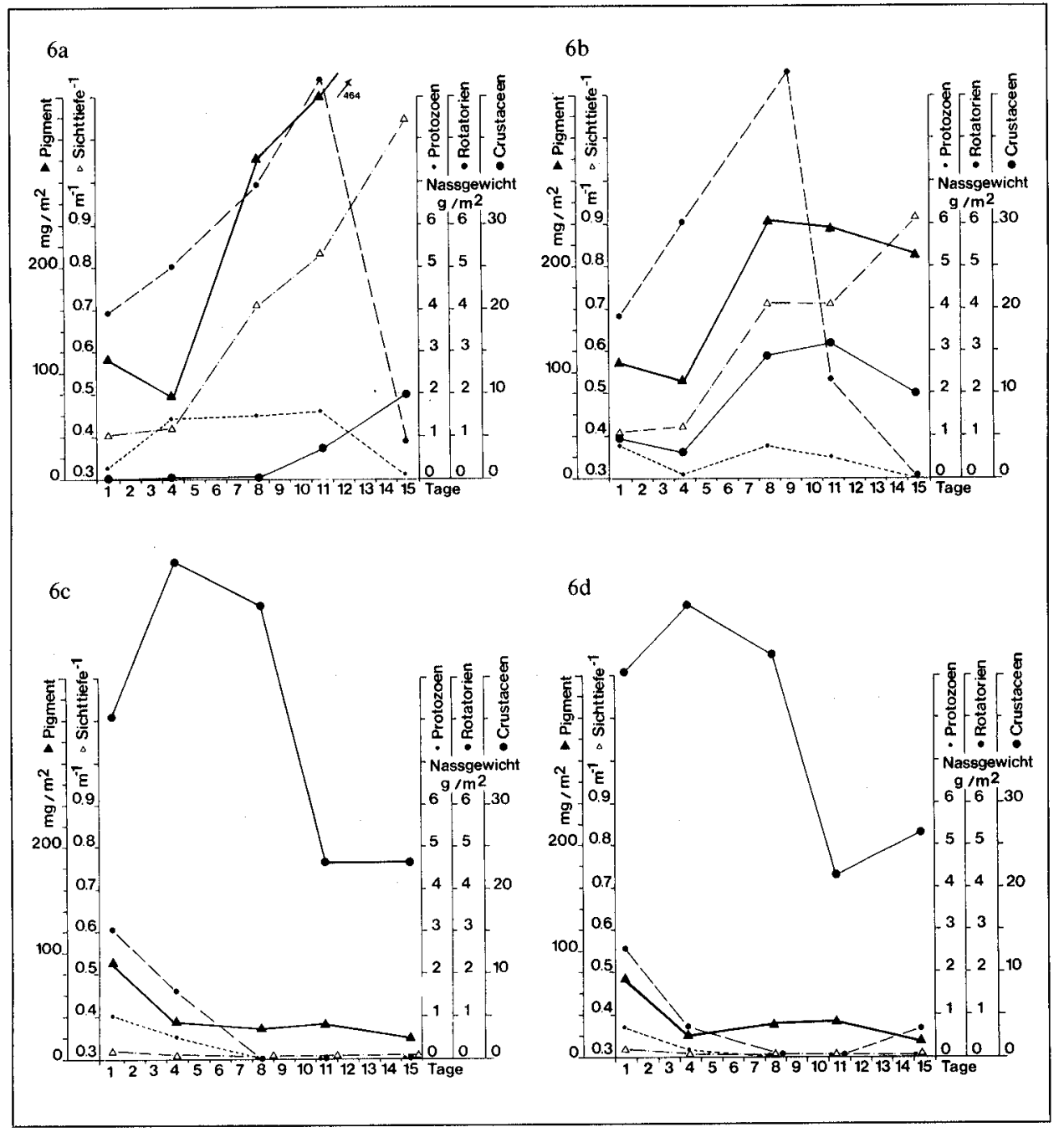

Abb. 6. Versuch III: Zeitreihen der Planktongruppen.

Figure 6. Experiment III: Succession of different planktonic groups.

Abb. 6a. Crustaceenplankton, $>95 \mu \mathrm{m}$, ausfiltriert.

Figure 6a. Planktonic crustaceans filtered out through a $95-\mu \mathrm{m}$ net.

Abb. 6b. Crustaceenplankton, $>200 \mu \mathrm{m}$, ausfiltriert.

Figure 6b. Planktonic crustaceans filtered out through a $200-\mu \mathrm{m}$ net.

Abb.6c. Biozönose, unverändert.

Figure 6c. Unaltered biocenosis.

Abb.6d. Crustaceenplankton, angereichert. Mittelwert aus zwei Parallelen.

Figure 6d. Enriched with planktonic crustaceans. Mean of two parallels. 


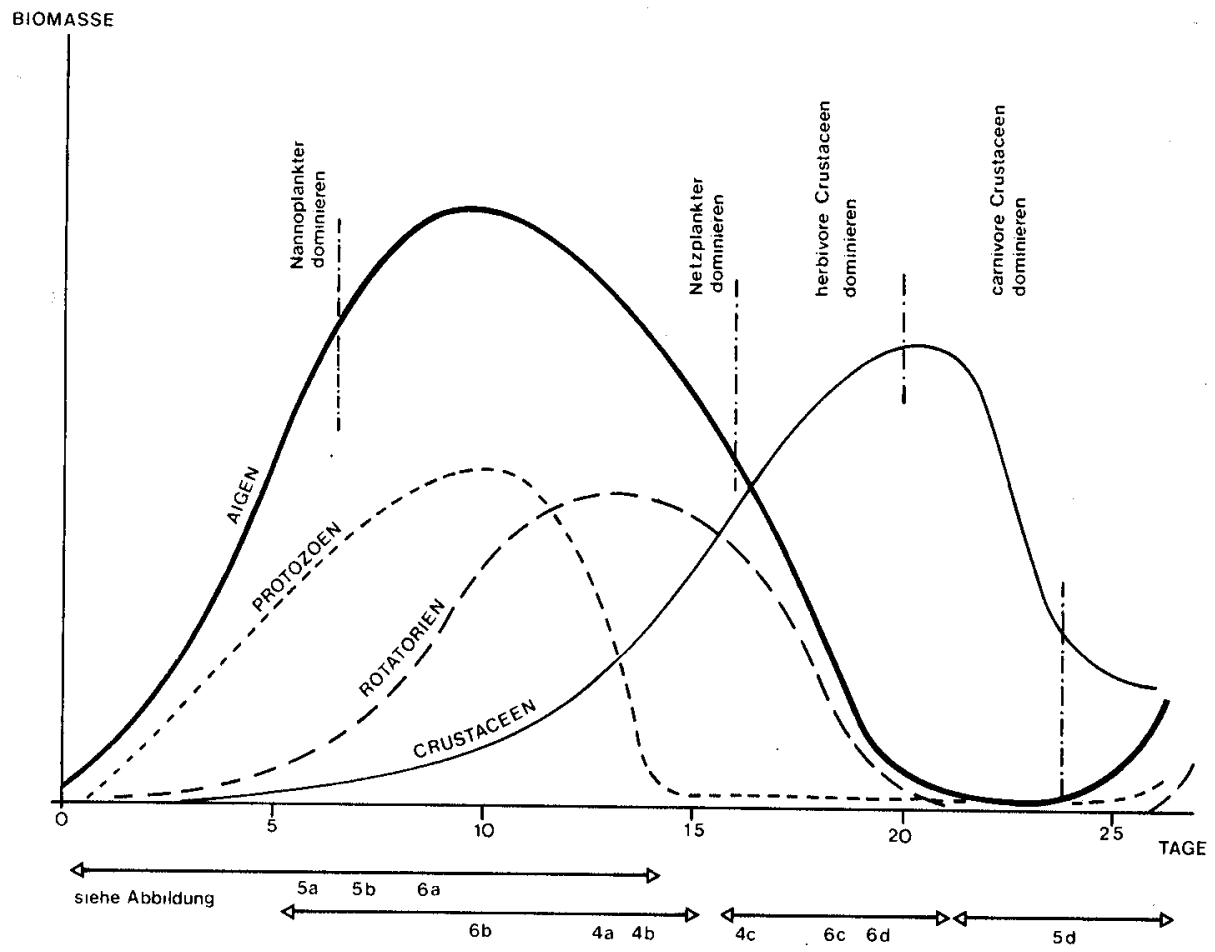

Abb. 7. Schematische Abfolge der Planktongruppen durch Verflechtung der Abb.4-6. Figure 7. Schematic sequence of the planktonic groups by means of coupling figures 4-6.

\subsection{Selektivität des Grazings}

Fütterungsversuche und Darmuntersuchungen an Crustaceen zeigen, dass die Nahrungsauswahl in erster Linie grössenselektiv ist $[4,17,42]$. Partikeln unter $20 \mu \mathrm{m}$ Durchmesser werden bevorzugt aufgenommen. Aufnahme und Verwertung grösserer Formen (z.B. Kolonien, Fäden, Kieselalgen) hängen wesentlich mit deren Zerbrechlichkeit zusammen. Da die systematischen Algengruppen häufig typische Zell- und Kolonieformen aufweisen, äussert sich eine grössen- oder formselektive Nahrungsaufnahme zwangsläufig auch in einer klassenspezifischen Selektion. Die aufgenommenen Algen werden unterschiedlich gut ausgenutzt. Die gleichzeitige Aufnahme von Algen ähnlicher Grösse und Form, aber unterschiedlicher Verwertbarkeit, deutet darauf hin, dass die Zooplankter in der Regel nicht in der Lage sind, die ihnen bekömmlichen Algen von gleich aussehenden, unverdaubaren zu unterscheiden oder zu trennen.

Die Algenbiozönose war in den drei hier beschriebenen Versuchen unterschiedlich aufgebaut. Die Sukzession, die sich unter der Einwirkung verschiedener Zooplank- 
tonpopulationen bei den Algen ergibt, wurde in Abb. 8 dargestellt. Das Plankton wurde in drei Grössenkategorien aufgeteilt:

Grosse Plankter (über $40 \mu \mathrm{m}$ Abmessung) umfassen Dinophyceen, Kieselalgen (pennate Formen), Blaualgenfäden, Jochalgen.

Mittelgrosse Plankter (10-40 $\mu \mathrm{m}$ Durchmesser) setzen sich aus zentrischen Kieselalgen, Cryptomonas-Arten und zahlreichen Chlorococcalen zusammen.

Als kleinere Algen (unter $10 \mu \mathrm{m}$ Durchmesser) wurden Rhodomonas, Kephyrion, Erkenia, $\mu$-Algen und kleine Flagellaten eingestuft.
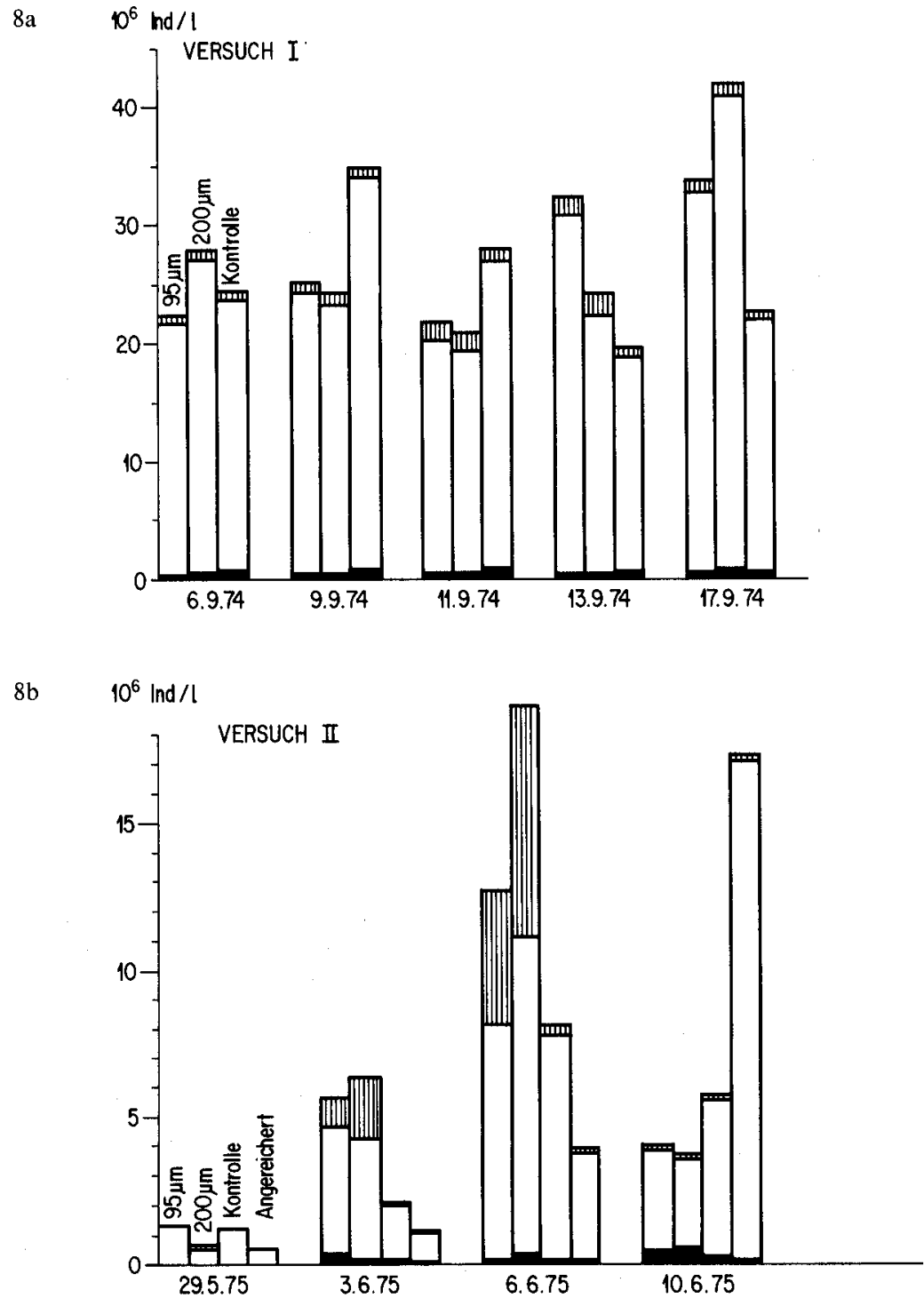

Abb. 8. Legende siehe Seite 52. 


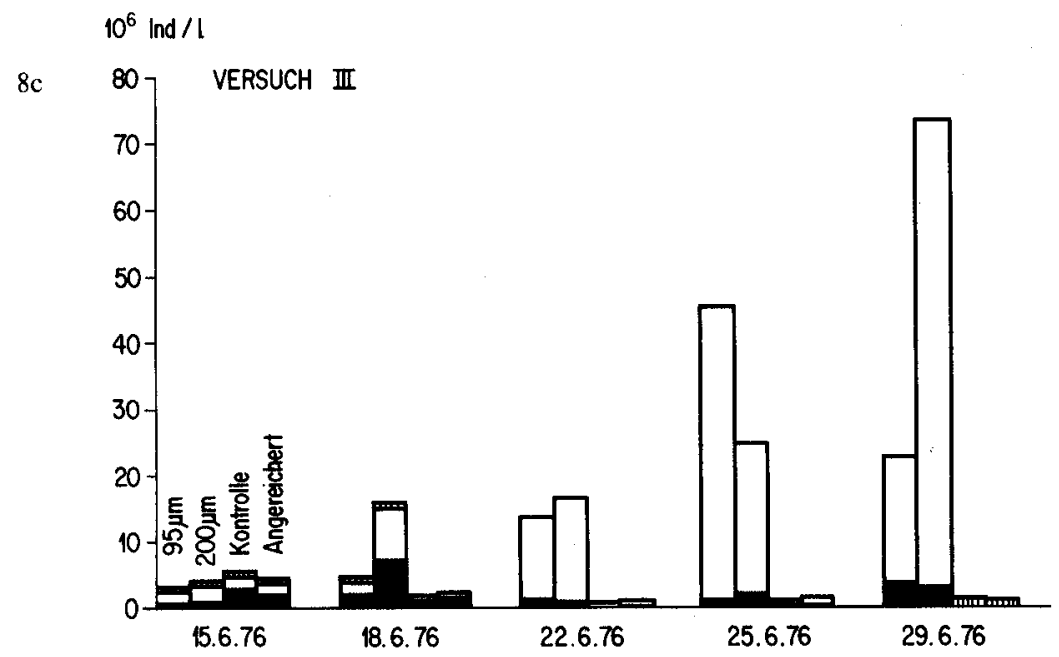

Abb. 8. Sukzession der Grössenfraktionen des Phytoplanktons. Gestreifte Balken: Individuendichte der Algen mit $<10 \mu \mathrm{m}$ Grösse. Weisse Balken: Individuendichte der Algen mit 10-40 $\mu \mathrm{m}$ Grösse. Schwarze Balken: Individuendichte der Algen mit grösseren Abmessungen. Es wurde stets die häufigste Form (z. B. Koloniegrösse) dargestellt.

Figure 8. Succession of the size fractions of the phytoplankton. Shaded portion: Density of algal individuals below $10 \mu \mathrm{m}$ in diameter. White portion: Density of algal individuals from 10 to $40 \mu \mathrm{m}$ in diameter. Black portion: Density of algal individuals with more than $40 \mu \mathrm{m}$ in diameter. Always the most abundant form was measured (e.g. colony size).

Das Planktonbild wird weitgehend durch den Anteil der mittelgrossen Algen dominiert. Kleine Formen können zwar sehr schnell hohe Individuendichten aufbauen, wenn sie nicht unterdrückt werden, fallen aber biomassemässig nicht ins Gewicht.

Das Ausfiltrieren der Crustaceen hat in den drei Versuchen unterschiedliche Resultate zur Folge. Im Versuch I erhöht sich die Individuendichte nur unbedeutend, während in den Versuchen II und III ein rasantes Wachstum der Algen einsetzt. Im Versuch II profitieren zunächst Mikroalgen und mittelgrosse Plankter von der Verringerung der Abweideaktivität, im Versuch III sind mittelgrosse und grosse Plankter bevorzugt. Hohe Rotatorien- und Protozoenpopulationen, welche in den filtrierten Einheiten verblieben, hinderten beim Versuch III die kleinsten Plankter in ihrer Entwicklung. Die Nährstoffe und das Lichtangebot werden hier von den grösseren Formen genutzt. Im Versuch II vermehren sich die kleineren Formen schneller und verdrängen die grossen Plankter vorerst. Mit dem Aufkommen der Protozoen und Rotatorien verschwinden die kleineren Formen schlagartig wieder, die freigesetzten Nährstoffe kommen nun wiederum den grossen Planktern zugute.

Die Dominanz von karnivoren Crustaceen verändert im Versuch III die Lebensbedingungen der Algen insofern, als die Filtrierer so weit dezimiert werden, dass 
kleine Algen - bei verringertem Grazing - dank hoher Teilungsrate am Schluss des Experiments die höchste Dichte aufweisen (Abb. 8c).

\subsection{Produktionsraten der Plankter}

Die Versuchsanordnung erlaubt die Abschätzung der Vermehrungsraten der häufigsten Phyto- und Zooplanktonarten unter natürlichen Bedingungen. Anhand der Entwicklungsdauer der Jugendstadien der Crustaceen lässt sich ferner die Erfolgsrate bestimmen, d.h. der Anteil der Tiere gleicher Generation, welche das adulte Stadium erreichen.

Die Verdopplungszeiten häufiger Plankter sind in Tabelle 3 aufgeführt. Die Tabelle enthält zum Vergleich einige Werte anderer Autoren, welche auf ganz verschiedenen Wegen über Chemostatmethode, Sedimentmessung, Produktionsmessung usw. die Verdopplungszeiten der Algen berechneten. In vielen Fällen ist recht gute

Tabelle 3. Verdoppelungszeiten wichtiger Plankter.

Table 3. Time for reproduction of the most abundant algae.

\begin{tabular}{|c|c|c|c|}
\hline Gattung, Art & $\begin{array}{l}\text { Verdopplungs- } \\
\text { zeit in Std. }\end{array}$ & $\begin{array}{l}\text { Gemessen in } \\
\text { Versuch Nr. }\end{array}$ & $\begin{array}{r}\text { Literaturwerte } \\
\text { für Verdopplungs- } \\
\text { zeit in Std. }\end{array}$ \\
\hline A nabaena flos-aquae & 40 & III & $27[14]$ \\
\hline Aphanizomenon flos-aquae & 36 & I & $12[40]$ \\
\hline Asterionella formosa & 31 & I & $12[24]$ \\
\hline Cyclotella sp. (klein) & 4,7 & II & $3-4[3]$ \\
\hline Fragilaria crotonensis & 26 & & 1) \\
\hline \multicolumn{4}{|l|}{ Melosira granulata var. } \\
\hline angustissima & 48 & I & \\
\hline Ceratium hirundinella & 75 & I & \\
\hline Peridinium cinctum & 50 & I & \\
\hline Ankyra ancora fo. spinosa & 15 & III & \\
\hline Chlamydomonas sp. & 8 & III & \\
\hline Ch, reinhardii & 7 & & {$[18]$} \\
\hline Coleastrum microporum & 22 & & ) \\
\hline Oocystis lacustris & 55 & III & 1) \\
\hline Pediastrum duplex & 43 & II & \\
\hline Phacotus lendneri & 11 & III & \\
\hline Scenedesmus quadricauda & 48 & II & \\
\hline \multicolumn{4}{|l|}{ Closterium acutum } \\
\hline var. variabile & 38 & II & \\
\hline Cosmarium sp. & 29 & II & \\
\hline Cryptomonas ovata/erosa & 16 & II & \\
\hline Rhodomonas lacustris & 8 & III & \\
\hline Keratella cochlearis & 30 & III & \\
\hline Polyarthra sp. & 24 & III & \\
\hline Strombidium viride & 24 & III & \\
\hline
\end{tabular}

1) Nach Bloesch [3] unter 24 Std.; nicht exakt bestimmt. 
Übereinstimmung zu beobachten. Die meisten Algen haben Verdopplungszeiten um ein bis zwei Tage. Algen mit sehr geringen Abmessungen weisen sehr oft kürzere Teilungsintervalle auf; am langsamsten wachsen die voluminösen Dinophyceen.

Die höchsten Werte der Primärproduktion wurden in den filtrierten Einheiten gemessen, und zwar mit $267 \mathrm{mg} \mathrm{C}_{\text {ass }} / \mathrm{m}^{3} \cdot \mathrm{h}$ (Versuch III). Bezieht man die Primärproduktion auf $1 \mathrm{mg}$ Pigment, sind die zooplanktonreichen Kulturen aber weit produktiver bei gleicher Lichtintensität. Bei Lichtsättigung war hier die Produktion pro mg Pigment $3,6 \mathrm{mg} \mathrm{C}_{\text {ass }} / \mathrm{m}^{3} \cdot \mathrm{h}$ gegenüber 1,1 und $1,4 \mathrm{mg} \mathrm{C}_{\text {ass }} / \mathrm{m}^{3} \cdot \mathrm{h}$ in den filtrierten Einheiten. Für diese Unterschiede kann nicht das Phosphatangebot verantwortlich sein, denn in allen Einheiten lag die aktuelle Konzentration über $30 \mu \mathrm{g} / \mathrm{l}$. Die dauernde Verjüngung der Algenkulturen durch hohe Grazing-Raten dürfte für diese bessere Lichtverwertung verantwortlich sein. Aber auch die Einwirkung von Exkreten (Vitamine, Wuchsstoffe) darf nicht ausser acht gelassen werden. Nach Porter [30] teilen sich Grünalgen, welche den Darm von Crustaceen unverdaut passierten, schneller als die gleichen Formen in zooplanktonfreien Kulturen.

Auch die Sekundärproduktion, speziell der Protozoen und Rotatorien, erreicht beachtliche Werte. Innerhalb von drei bis vier Tagen konnte oft eine Verzehnfachung des Rotatorien- und Protozoenbestandes festgestellt werden. Dies entspricht einer Verdopplungszeit von etwa einem Tag (siehe Tab.3). Berücksichtigt man eine Sterberate von 10\%/Tag [32] und die Predationsverluste (variabel), gelangt man zu Verdopplungszeiten von $<20$ Std.

Der exponentielle Anstieg der Protozoen und Rotatorien folgt auf eine deutliche «lag-phase» (Abb. 5a, c, d). Da die Vermehrung der Protozoen auf der mitotischen Zellteilung beruht und die häufig vorkommenden Rotatorien (Keratella cochlearis, K. quadrata) meist nur ein Ei mit sich tragen, lässt der rasante Anstieg auf eine hohe Erfolgsrate schliessen. Potentielle Räuber dieser Zooplankter können offenbar bei diesem rasanten Biomassezuwachs nicht mithalten. Die Versuchsdauer von $14 \mathrm{Ta}$ gen reichte allerdings nicht aus, um einen vollständigen Vermehrungszyklus der Crustaceen zu erfassen. Die Produktionsrate (gemessen wurde hier der Biomassezuwachs) lag im besten Fall in der Grössenordnung von 30 bis 50\%/Tag. Sie war in den filtrierten Einheiten besser als in den zooplanktonreichen Kulturen.

Nach Metz [25] weisen Jugendstadien der Crustaceen eine höhere Sekundärproduktion pro konsumierte Nahrungseinheit auf als adulte Tiere. Die besondere Altersstruktur der Crustaceen in den filtrierten Einheiten (praktisch keine adulten Tiere) und die damit verbundenen geringen Predationsverluste waren für die erhöhte Sekundärproduktion in den filtrierten Testeinheiten verantwortlich.

Die Erfolgsrate der Copepoden entsprach diesen Befunden: In den filtrierten Einheiten erreichten $87-96 \%$ der Nauplien das Copepodidstadium im Laufe von 6 bis 10 Tagen. Von den anfänglich vorhandenen Copepodiden werden mehr als $90 \%$ geschlechtsreif. Gegenüber Aufzuchtversuchen von Mittelholzer [26] (unter Ausschluss der Predation) ergibt das eine um rund 20\% bessere Erfolgsrate, was mit der grösseren Nahrungsdiversität in unsern Versuchsbehältern zusammenhängen dürfte. Die hohen Predationsverluste in den zooplanktonreichen Kulturen lassen die Erfolgsrate auf Werte unter 30\% sinken. In einigen Fällen erreichen weniger als 20\% der Nauplien das Copepodidstadium. Die adulten Cyclops leben offenbar zu einem beachtlichen Teil kannibalisch von ihren eigenen Nachkommen. Die Produktions- 
und Erfolgsraten der Daphnien sind weit schlechter bekannt. Regelmässig werden gegen Versuchsende infolge Nahrungsmangel Ephippien gebildet. Bei optimaler Fütterung bei $20^{\circ} \mathrm{C}$ erreichen Daphnien in Kultur eine Gewichtszunahme der Gesamtpopulation (schliesst die Vermehrung mit ein) von 30\% [9]. In den zooplanktonreichen Kultureinheiten nehmen die Crustaceen an Dichte ab, da die Predation und die Sterberate grösser sind als die Produktionsrate.

\subsection{Freisetzung und Sedimentation der Nährstoffe}

Die Sedimentation der Nährstoffe hängt in erster Linie von der Algendichte $a b$ (Abb.9), doch tragen auch tote Zooplankter einen geringen Teil zur Sedimentation bei. Bei Annahme einer 10tägigen Lebensdauer für Rotatorien und einer 20tägigen Lebensspanne für Crustaceen ergäbe das bei gleichbleibender Populationsdichte ohne anderweitige Verluste etwa 7\% der aktuellen Zoobiomasse pro Tag.

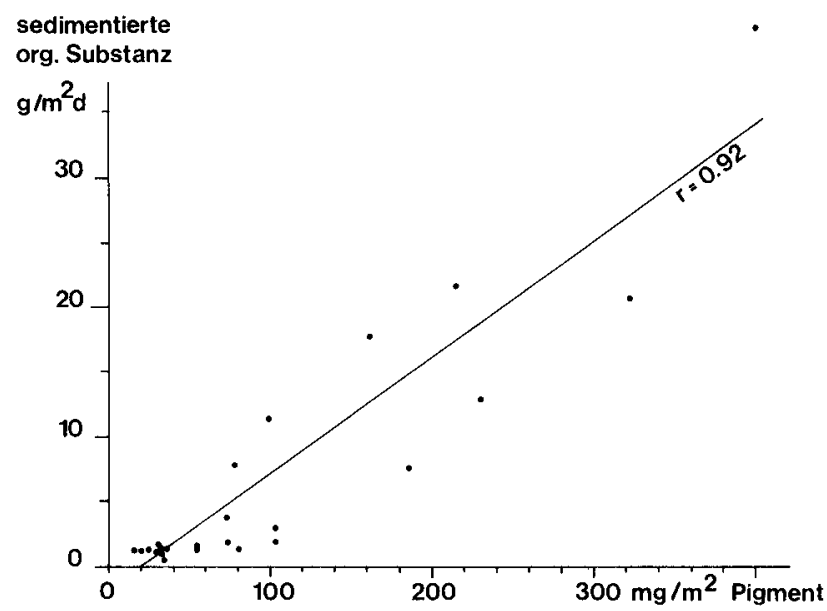

Abb. 9. Abhängigkeit der Sedimentation organischer Substanz von der überstehenden Algenbiomasse (Mass für die Algenbiomasse: Pigmentgehalt).

Figure 9. Sedimentation of organic substance dependent on the algal standing crop (expressed as pigment content).

Die intensive Predation verhindert aber sehr oft das Erreichen des Alters und einen natürlichen Alterstod im Plankton. Im gleichen Sinne verringern hohe GrazingRaten die Inkorporation der Nährstoffe in Algenbiomasse und deren Sedimentation.

Bei geringen Herbivorenbeständen fällt pro assimilierte Kohlenstoffeinheit mehr als doppelt soviel Sediment (Trockengewicht) an als in zooplanktonreichen Kulturen 
(Abb.10). Während intensiver Grazing-Phasen wird weit mehr abgeweidet, als in Zooplanktonbiomasse angelegt wird. Dementsprechend wird die Rezirkulation der Nährstoffe gefördert. Führt der Abweideverlust zu einer Algendepression (Overgrazing), dann kommt es zu einer Akkumulation der Nährstoffe im Epilimnion. Das System ist in diesem Moment limitiert durch die tief gehaltene Algendichte, welche das Nährsubstrat nicht nutzen kann. Der Verlauf der Phosphatkonzentration (Abb.11) zeigt in den zooplanktonreichen Kulturen eine deutliche Phosphorfreisetzung durch Grazing.

Vollständiges Ausbleiben des Grazings äusserst sich umgekehrt in einer rapiden Produktionsphase des Phytoplanktons bis zur nahezu vollständigen Inkorporation

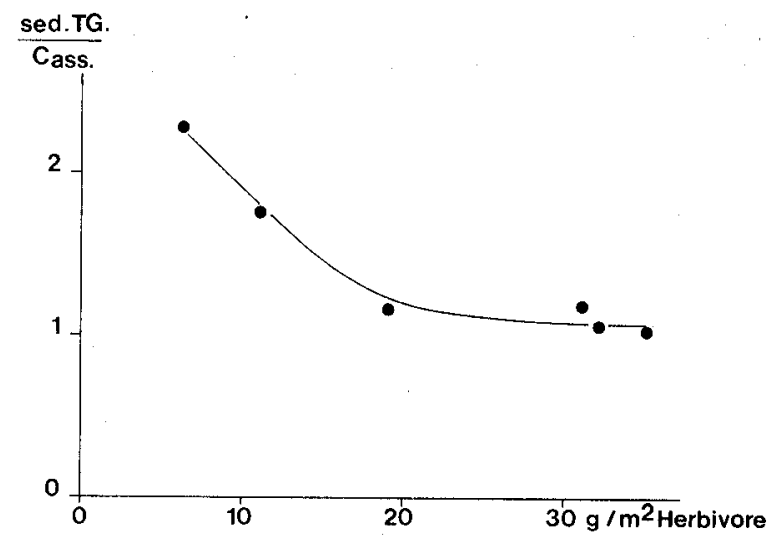

Abb. 10. Abhängigkeit der Sedimentationsrate von der Herbivorendichte. Figure 10. Sedimentation rate dependent on the density of the herbivores.

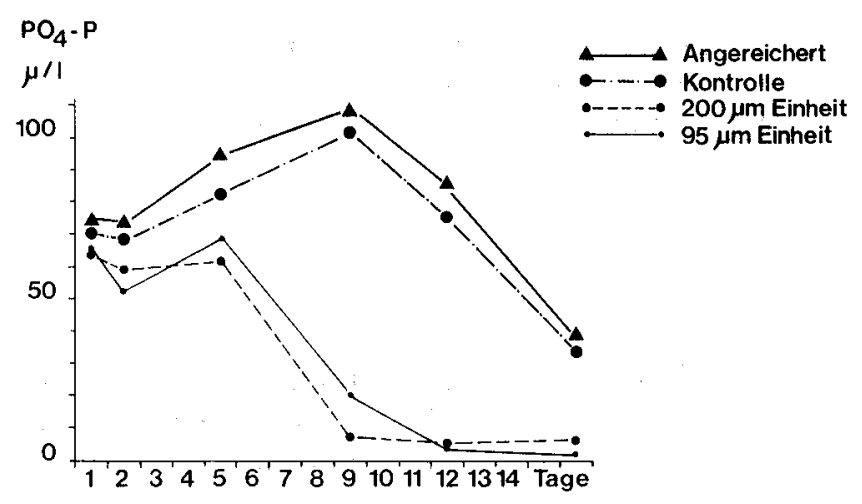

Abb. 11. Dynamik der Phosphatkonzentration in den Kultureinheiten (Versuch III). Figure 11. Dynamics of the phosphate concentration in the culture units (experiment III). 
des verfügbaren Phosphats. Die geringen Phosphatkonzentrationen fördern das Aufkommen von Algen, z. B. von pennaten Kieselalgen, welche mit den gespeicherten Nährstoffen sehr haushälterisch umgehen [15]. Die Anfangs- und Endwerte der Phosphatkonzentrationen der verschiedenen Versuchseinheiten weisen auf die unterschiedliche P-Zehrung hin (Tab.4).

Tabelle 4. Phosphatkonzentrationen in $\mu \mathrm{g} / 1 \mathrm{zu}$ Beginn und am Schluss der Versuche.

Table 4. Concentration of phosphate at the beginning and the end of the experiments.

\begin{tabular}{lllcclr}
\hline Kultureinheit & $\begin{array}{l}\text { Versuch I } \\
\text { Beginn }\end{array}$ & Ende & $\begin{array}{l}\text { Versuch II } \\
\text { Beginn }\end{array}$ & Ende & $\begin{array}{l}\text { Versuch III } \\
\text { Beginn }\end{array}$ & Ende \\
\hline $95-\mu \mathrm{m}$-Einheit & $5,6 \pm 0,4$ & $3,6 \pm 1,0$ & 97 & 11 & 64 & 2 \\
$\begin{array}{l}\text { Ko0- } \mu \text { m-Einheit } \\
\text { Kontrollen }\end{array}$ & $6,0 \pm 0,2$ & $3,6 \pm 1,5$ & 100 & 9 & 65 & 6 \\
$\begin{array}{l}\text { Angereichert mit } \\
\text { Zooplankton }\end{array}$ & Keine Einheit & 100 & 36 & 70 & 33 \\
\hline
\end{tabular}

Für die Aufrechterhaltung einer Zooplanktonbiomasse ist die stete Nachlieferung organischer Energie nötig. Die Zooplankter haben keine Veranlassung, die für die Pflanze so wichtigen Nährstoffe über das Mass der stöchiometrischen Plasmazusammensetzung hinaus zu speichern. Die Algen hingegen neigen zu einem Luxuskonsum der Nährstoffe [15]. Blum [5] und Smith [37] konnten zeigen, dass Algen schon in sehr kurzer Zeit (<30 Minuten) über $75 \%$ vom zusätzlich angebotenen Phosphat aufgenommen und im Zellplasma in organischer Verbindung gespeichert haben.

Durch die Freisetzung des Phosphats in den zooplanktonreichen Kulturen wird nun dieser Luxuskonsum gefördert, und die Algenbiozönose wechselt auf Arten, welche bei hohen P-Gehalten besser wachsen können [10].

Das P:C-Verhältnis der Algen und damit auch des Sediments wird durch die schnelle Rezirkulation des Zooplanktons erhöht. Die wenigen Daten unserer Versuche deuten darauf hin, dass der P-Gehalt der organischen Substanz im Sediment stark von der Aktivität des Zooplanktons im überstehenden Wasser abhängt. Das Sediment der zooplanktonreichen Kulturen wies 3,8\% P-Anteil auf gegenüber von $1 \%$ in filtrierten Einheiten.

\section{Diskussion}

Anhand von Sedimentationsmessungen und Untersuchungen über den Phosphorhaushalt der Gewässer folgern Bloesch [3] und Gächter [10], dass die gemessene Primärproduktion nur durch einen wirkungsvollen kleinen epilimnischen Nährstoffkreislauf sichergestellt werden kann. Durch Wechselwirkungen der Zooplankter mit dem Phytoplankton wird dieser Kreislauf beeinflusst. Im Jahresverlauf ändern sich die Populationen von Phytoplankton und Zooplankton dauernd. Der Verlauf der Algen und Herbivoren gleicht in gewissen Abschnitten einer Räuber-Beute-Relation, wie sie in den Volterraschen Gesetzen formuliert ist. Durch Bildung von Dauerstadien $\left(\mathrm{C}_{4}\right.$-Copepodiden, welche eine Diapause einschalten, oder Ephippien) 
sind aber Elemente vorhanden, welche die Entwicklung der Biozönose entgegen der erwarteten Räuber-Beute-Beziehung in eine andere Richtung führen [7, 22]. Die ungleich lange Generationszeit von Räuber- und Beuteorganismen ist der Grund für ihre zeitlich phasenverschobenen Wachstumskurven. Untersuchungen im Bodensee haben gezeigt, dass die meistens im Mai-Juni registrierten "Klarwasserstadien» durch hohe Abweideverluste hervorgerufen werden. Dieser Zäsur in der Algenentwicklung folgt häufig auch eine Verschiebung in der qualitativen Zusammensetzung der Algenbiozönose. Vor dem Zusammenbruch dominieren zentrische Kieselalgen und kleine Flagellaten (Cryptophyceen), nach der intensiven Abweidephase setzt sich das Plankton aus grösseren Planktern der Klassen Diatomeae und Conjugatophyceae zusammen [7]. Unsere Experimente zeigen übereinstimmend mit Porter [29-31], dass die Algensukzession in hohem Masse von der zur Versuchszeit starken Zooplanktonpopulation abhängt. Neben den spezifischen Abweideverlusten spielen möglicherweise noch weitere, hier nicht behandelte Selektionsmechanismen (Sedimentation, Toxinproduktion, Parasitenbefall usw.) eine Rolle in der Ablösung der Algenarten.

Es wäre verfehlt, die Algensukzession nur als Resultat der Selektion zu betrachten, wirken doch zahlreiche andere Faktoren und nicht zuletzt endogene Eigenschaften (z. B. Bildung und Keimung von Dauerzysten, Toleranz gegenüber Änderungen usw.) auf eine periodische Ablösung der Arten hin. Die Selektivität des Grazings ist aber mindestens in der Hauptvegetationsperiode nicht zu vernachlässigen.

Entsprechend den momentanen Aktivitäten und Räuber-Beute-Relationen können die Nährstoffe zur Synthese einer grösseren oder kleineren Biomasse genutzt werden. Ohne Zooplankton sedimentiert ein grösserer Anteil der Primärprodukte, aber mit geringerem P-Gehalt. Mit Zooplankton ist die Sedimentationsrate herabgesetzt, die Aktivität der Algenzellen und deren P-Gehalt jedoch erhöht. Für einen See, der an Überdüngung leidet, ist die unter der Einwirkung des Zooplanktons ineffiziente P-Nutzung günstiger, da weniger organische Substanz pro Phosphor das Hypolimnion erreicht.

Die kleineren Formen des Zooplanktons sind auf die Nachlieferung hoher Algendichten von Ultraplanktern angewiesen. Trotz beachtlicher Biomassezunahme verursachen sie nur geringfügige Verluste bei der Algenbiomasse. Gegenüber gleich starken Zooplanktonbiomassen herbivorer Crustaceen ist die Grazing-Aktivität viel geringer. Das deutet aber auf eine erheblich bessere Verwertung der konsumierten Nahrung durch Protozoen und Rotatorien hin, wofur die verschiedenartigen Verdauungssysteme verantwortlich sein dürften. In Nahrungsvakuolen oder blind endenden Darmsystemen dürfte die Assimilation der organischen Substanz vollständiger sein als im kurzen, geraden Darm von Phyllopoden. Dies gilt insbesondere für Verhältnisse mit hoher Algendichte, wo durch die Filtriertätigkeit, welche nicht unter eine von der Sauerstoffversorgung bestimmte Aktivität absinken kann, der Nahrungsbrei zu schnell durch den Darm befördert wird, um verdaut zu werden [27].

Die geringen Bestände an Rotatorien und Protozoen reichen bei den beobachteten Verdopplungszeiten aus, um in kürzeren Perioden mit günstigen Wachstumsbedingungen zu hohen Biomasse-Erträgen zu kommen. In unseren Experimenten folgen die Peaks dieser Organismen nur einige Tage hinter den Algenspitzen nach. Bei 
grösseren Probenahmenintervallen lässt sich diese zeitliche Verschiebung nicht mehr erkennen. Die Algenspitzen fallen dann mit den Maxima der Rotatorien zusammen [7]. Die Entwicklungsmaxima der Crustaceen jedoch folgen erst mit einer Verzögerung von 14 Tagen und mehr hinter den Algenmaxima nach.

Durch Zusammensetzen der einzelnen Entwicklungsgänge, entsprechend den Startbedingungen der verschiedenen Kultureinheiten, ergibt sich eine schematische Sukzession der Planktonkompartimente, wie sie bei einer Erstbesiedelung eines nährstoffreichen Biotops (mit allseitiger Impfung) zu erwarten wäre (Abb.7). Die Entwicklungsgänge von Protozoen, Rotatorien und Crustaceen weisen einige feine Unterschiede auf. Die Protozoen wachsen praktisch simultan mit den Algen, während Rotatorien und Crustaceen zunächst eine längere Anlaufphase erleben. Durch die Konkurrenz der wachsenden Rotatorien und Crustaceen bricht für die Protozoen, welche fast vollständig von Nannoplanktern und Bakterien leben, die Nahrungsgrundlage sehr schnell zusammen. Sie werden aber zunehmend auch von den andern Zooplanktern durch Predation zahlenmässig reduziert. Bei den Rotatorien und Crustaceen findet ein Ablöseprozess statt, der zu erhöhten Räuberbeständen führt.

Den Crustaceen und Rotatorien kommt eine grosse Diversität der Algen eher zugute, da die Nahrungsbedürfnisse nicht so eng begrenzt sind wie bei Protozoen. Der Rückgang der Rotatorien geht daher weniger schlagartig vor sich, und bei den Crustaceen bilden sich bei hohen Beständen relativ stabile Relationen herbivorer und karnivorer Crustaceen aus. Die räuberischen Copepoden vermehren sich nicht bis zur vollständigen Depression der herbivoren Zooplankter. Da die Jugendstadien von Cyclopsarten auch zum Teil herbivor leben, wird die Räuberlarve durch ihre spätere potentielle Beute, die viel effektiver filtrierenden Blattfusskrebse oder Rotatorien, konkurrenziert. Da die räuberischen Cyclops auch Copepodiden ergreifen, reguliert sich die Räuberpopulation bis zu einem gewissen Grade selbst.

Entsprechend den Veränderungen der Planktonbiozönose variieren auch die Stoffflüsse zwischen den Kompartimenten. Die Antwort der Biozönose auf einen bestimmten Nährstoff- bzw. Energie-Input kann daher je nach der Zusammensetzung der aktuellen Biozönose ganz verschieden ausfallen. Die Änderungen der Produktion und Sedimentation durch Variation der Herbivorendichte können sich in der Grössenordnung einer halben Zehnerpotenz bewegen.

\section{Zusammenfassung}

1. Mit Planktonnetzen (Maschenweite $95 \mu \mathrm{m}$ und $200 \mu \mathrm{m}$ ) wurde das Plankton in 25001 fassenden Kultureinheiten in situ grössenselektiv verändert. Es wurden die Effekte der Ausfiltration bzw. der Anreicherung von Crustaceen studiert.

2. Wurden die Crustaceen quantitativ entfernt, vermehrten sich die Algen sprunghaft und erreichten innerhalb von 10 Tagen eine Verzehnfachung der Anfangsbiomasse. Die Verdopplungszeiten einzelner Algenarten bewegten sich zwischen 4 Stunden und 3 Tagen.

3. Unmittelbar auf die Algenmaxima folgten hohe Biomassen von Protozoen. Etwa 2-3 Tage phasenverschoben entfalteten sich die Rotatorien massenhaft. Crustaceen folgten erst nach einer längerdauernden «lag-phase». Die Verdoppelungszeit der 
Zooplanktonbiomasse bewegte sich für die kleineren Formen (Protozoen, Rotatorien) in der Grössenordnung von einem Tag; für Crustaceen waren 2-3 Tage für eine Verdoppelung nötig.

4. Die einzelnen Zooplanktongruppen weideten die Algenbiomasse unterschiedlich, d.h. grössenselektiv, ab. Die Entfaltung der Protozoen war mit keiner signifikanten Depression der Algenbiomasse verknüpft. Rotatorien und Crustaceen bevorzugten Nannoplankter; diese wurden dadurch deutlich dezimiert. Bei gleicher Zooplanktonbiomasse (Rechenvolumina) weiden Crustaceen mehr Algen ab pro Zeiteinheit als Rotatorien und Protozoen. Die kleineren Formen konnten die Nahrung besser verwerten.

5. Die Zooplanktongruppen beeinflussen sich gegenseitig, nämlich direkt durch Predation (Protozoen werden von Rotatorien und diese beide von Crustaceen unterdrückt) und indirekt durch Nahrungskonkurrenz. Die Anreicherung der Zooplankter begünstigt stets den Räuber durch Verkürzung der Distanz zur Beute und dadurch erhöhte Begegnungshäufigkeit.

6. Die Zooplanktondichte beeinflusste die Sedimentationsrate. Hohe Herbivorenbestände verringern die Sedimentationsrate der organischen Substanz; diese weist aber ein höheres P:C-Verhältnis auf als das Sediment aus zooplanktonfreien Kultureinheiten.

7. In Phasen des Overgrazings wurden die gelösten Komponenten der Pflanzennährstoffe erhöht, was den Luxuskonsum des Phosphors durch die verbliebenen Algen förderte.

\section{SUMMARY}

1. Through filtering with plankton nets (mesh size $95 \mu \mathrm{m}$ and $200 \mu \mathrm{m}$ ) the plankton was selected according to size in $2500 \mathrm{l}$ culture units in situ. The effects of exclusion or enrichment of crustacea were studied.

2. Quantitative removal of crustacea caused a rapid development of algae and a tenfold increase of the initial biomass within 10 days. Generation times of some algal species ranged between $4 \mathrm{~h}$ and 3 days.

3. A direct consequence of algal maxima was the development of high biomasses of protozoa. Approximately 2-3 days later, a massive growth of rotatoria was also encountered. Crustacea followed only after a longer lag phase. Generation time of zooplanktonic biomass was for smaller forms (protozoa, rotatoria) in the order of one day; crustacea required 2-3 days to double the biomass.

4. Grazing of algal biomass varied among the individual zooplankton groups, i.e. size-selective consumption was identified. The development of protozoa did not reduce the algal biomass significantly. Nannoplankton were clearly decimated since rotatoria and crustacea favoured them. Experiments with an equal quantity of zooplanktonic biomass (calculated volume) showed that crustacea graze more algae per unit of time than rotatoria and protozoa. The food was more efficiently utilized by smaller forms.

5. The zooplanktonic groups influence each other, namely directly by predation (protozoa are suppressed by rotatoria and both of these in turn get suppressed by crustacea) and indirectly by food competition. Enrichment of zooplankton is always beneficial to the predator since it shortens the distance to the prey and raises the probability of chance encounters.

6. The density of the zooplankton influenced the sedimentation rate. Large herbivorous populations reduce the rate of sedimentation of organic substance; its $\mathrm{P}: \mathrm{C}$ ratio, however, is higher than that of the sediment of culture units free from zooplankton.

7. During overgrazing phases more soluble components of plant nutrients are released, thus promoting luxury consumption of phosphorus by the remaining algae. 


\section{RÉSUMÉ}

1. Pour étudier l'effet d'une exclusion ou de l'enrichement des crustacés les organismes planctoniques furent filtrés en unités de culture de 25001 contenu (largeur de mailles $95 \mu \mathrm{m}$ et $200 \mu \mathrm{m}$ ).

2. Après l'exclusion complète des crustacés les algues se multiplièrent rapidement et atteindraient en 10 jours une biomasse dix fois autant du début. Le temps de redoublement de quelques espèces fut entre 4 heures et 3 jours.

3. Immédiatement après les maxima d'algues un développement massif de protozoaires suivit. Après 2 ou 3 jours les rotifères se développèrent fortement. Les crustacés apparurent après une longue lag-phase. Le temps de redoublement de la biomasse zooplanctonique fut pour les formes petites (protozoaires, rotifères) au milieu un jour et pour les crustacés $2-3$ jours.

4. Les différents groupes du zooplancton se repurent différemment des algues selon les dimensions. Le développement des protozoaires ne fut pas lié à une dépression significative de la biomasse d'algues. Les rotifères et les crustacés choisirent le nannoplancton. Dans une biomasse équivalente les crustacés se repurent plus fort des algues que les rotiferes et les protozoaires. Les petites formes tiraient mieux profit de la nourriture que les grandes formes.

5. Les différents groupes du zooplancton s'influencent réciproquement; directement par la prédation (les protozoaires sont supprimées par les rotifères et les deux sont supprimées par les crustacés), et indirectement par la concurrence d'alimentation. Après l'enrichement du zooplancton le prédateur est favorisé par la distance réduite entre prédateur et nourriture.

6. La densité du zooplancton influence la sédimentation. Des organismes hérbivores en grand nombre diminuent la sédimentation de matières organiques; leur proportion P:C est plus haut qu'au sédiment dans les unités de culture sans zooplancton.

7. Les components dissous des substances nutritives sont présents en grand nombre dans les situations de l'overgrazing ce qui hâta la consommation superflue du phospor par les algues restantes.

\section{VERDANKUNG}

An den Arbeiten waren beteiligt: Herr Heinz Bachmann (Exposition und Konstruktion der Versuchseinheiten), Frl. Beatrix Egli (Bestimmung und Zählung der Rotatorien und Crustaceen), Frau Dr. Doris Fastrich (Auszählung der Phytoplankter und Protozoen), Herr Bruno Ribi und Herr Richard Illi (Pigmentbestimmung und chemische Analytik). Bei der Probenahme wirkten Doktoranden und Studenten im Rahmen des Biologiepraktikums «Aquatische Ökologie» mit. Die Durchsicht des Manuskripts besorgte Prof. H. Ambühl. Für die zahlreichen Hilfestellungen möchten wir allen Beteiligten herzlich danken.

\section{LITERATURVERZEICHNIS}

1 Ambühl, H., und Schmid, M.: Bestimmung geringster Mengen von Phosphation im Wasser von Binnenseen. Schweiz. Z. Hydrol. 27, 171-183 (1965).

2 Anderson, G.C., Comita, G.W., und Engstron-Heg, V.: A Note on the Phytoplankton-Zooplankton Relationships in two Lakes in Washington. Ecology 36, 757-759 (1955).

3 Bloesch, J.: Sedimentation und Phosphorhaushalt im Vierwaldstättersee (Horwer Bucht) und im Rotsee. Schweiz. Z. Hydrol. 36, 71-186 (1974).

4 Burns, C.W.: Particle Size and Sedimentation in the Feeding Behavior of two Species of Daphnia, Limnol. Oceanogr. 14, 393-402 (1969).

5 Blum, J.J.: Phosphate Uptake by Phosphate Starved Euglena. J. Physiol. 49, 1125-1137 (1966).

6 Bürgi, H.R.: Die Wirkung von NTA auf das Wachstum des Phytoplanktons unter besonderer Berücksichtigung des Eisens als Mikroelement. Schweiz. Z. Hydrol. 36, 1-70 (1974).

7 Bürgi, H.R.: Die langjährige Entwicklung des Phytoplanktons im Bodensee (1963-1973); Teil I: Untersee. Ber. int, Gewässerschutzkomm. Bodensee $2 I$ (1977).

8 Edmondson, W.T.: Food Supply and Reproduction of Zooplankton in Relation to Phytoplankton Population. Rapp. P.-v. Réun. Cons. perm. int. Explor. Mer 153 (1962).

9 Fricker, H.J.: Beeinflussung der Phytoplankton-Aktivität durch Zooplankton-Populationen bei verschiedenen Nährstoffbedingungen (in Vorbereitung). 
10 Gächter, R.: Phosphorhaushalt und planktische Primärproduktion im Vierwaldstättersee (Horwer Bucht). Schweiz. Z. Hydrol. 30, 1-66 (1968).

11 Gächter, R.: Die Bestimmung der Tagesraten der planktischen Primärproduktion. Modelle und Insitu-Messungen. Schweiz. Z. Hydrol. 34, 211-244 (1972).

12 Goldman, Chr.R.: A Method of Studying Nutrient Limiting Factors in situ in Water Columns by Polethylene Film. Limnol. Oceanogr. 7, 99-101 (1962).

13 Goltermann, H. L.: Chemical Analysis of Fresh Waters. IBP-Handbook Nr. 8 (1969).

14 Gorham, P. R., in: The Biology of Blue-green Algae. Hrsg. Carr, N.G., und Whitton, B.A. Blackwell Scientific Publications, Oxford 1973.

15 Grim, J.: Der Phosphor und die pflanzliche Produktion im Bodensee. GWF 108, 1261-1271 (1967).

16 Haney, J.F.: An in situ-Examination of the Grazing Activities of Natural Zooplankton Communities. Arch. Hydrobiol. 72, 87-132 (1973).

17 Hargrave, B.T., und Green, G.H.: Effects of Copepod Grazing on two Natural Phytoplankton Populations. J. Fish. Res. Bd Canada 27, 1395-1403 (1970).

18 Hoogenhout, H., und Amesz, J.: Growth Rates of Photosynthetic Microorganisms in Laboratory Cultures. Arch. Hydrobiol. 50, 10-25 (1965).

19 Imboden, D.M., und Gächter, R.: A Dynamic Lake Model for Trophic State Prediction. Ecol. Modelling 4, 77-98 (1978).

20 Infante, A.: Untersuchungen über die Ausnutzbarkeit verschiedener Algen durch das Zooplankton. Arch. Hydrobiol., Suppl. 42, 340-405 (1973).

21 Krogh, A., und Berg, K.: Über die chemische Zusammensetzung des Phytoplanktons aus dem Frederiksborg-Schloßsee und ihre Bedeutung für die Maxima der Cladoceren. Int. Revue ges. Hydrobiol. Hydrogr. 25 (1931).

22 Lampert, W., und Schober, U.: Das regelmässige Auftreten von Frühjahrs-Algenmaximum und «Klarwasserstadium» im Bodensee als Folge von klimatischen Bedingungen und Wechselwirkungen zwischen Phyto- und Zooplankton. Arch. Hydrobiol. 82, 364-386 (1978).

23 Lantzsch, K.: Studien über das Nannoplankton des Zugersees und seine Beziehung zum Zooplankton. Z. wiss. Zool. 108 (1914).

24 Lund, J.W.G.: Studies on Asterionella. I. The Origin and Nature of the Cells Producing Seasonal Maxima. J. Ecol. 37, 389-419 (1949).

25 Metz, H.: Kohlenstoffinhalt, Aufnahme und Abgabe des Kohlenstoffs bei Daphnia pulex. Arch. Hydrobiol., Suppl. 42, 232-252 (1973).

26 Mittelholzer, E.: Populationsdynamik und Produktion des Zooplanktons im Greifensee und im Vierwaldstättersee. Schweiz. Z. Hydrol. 32, 90-149 (1970).

27 Monakov, A. V.: Review of Studies on Feeding of Aquatic Invertebrates Conducted at the Institute of Biology of Inland Waters, Academy of Science, USSR. J. Fish. Res. Bd Canada 29, 363-383 (1972).

28 Nauwerck, A.: Die Beziehungen zwischen Zooplankton und Phytoplankton im See Erken. Symb. bot. upsal. 17/5, 1-163(1963).

29 Porter, K.G.: A Method for the in situ Study of Zooplankton Grazing Effects on Algal Species Composition and Standing Crop. Limnol. Oceanogr. 17, 913-917 (1972).

30 Porter, K. G.: Selective Grazing and Differential Digestion of Algae by Zooplankton. Nature 244, 179-180 (1973).

31 Porter, K.G.: Beziehungen zwischen Pflanzen und Tieren in Süsswasser-Ökosystemen. Am. Scient. 65, 159-170 (1977).

32 Ruttner-Kolisko, A.: Rotatoria. In: Die Binnengewässer 26, Teil 1. Schweizerbartsche Verlagsbuchhandlung, Stuttgart 1972.

33 Ryther, J.H.: Inhibitory Effects of Phytoplankton Upon Feeding of Daphnia magna with Reference to Growth, Reproduction and Survival. Ecology 35, 522-533 (1954).

34 Schindler, J.E.: Food Quality and Zooplankton Nutrition. J. Anim. Ecol. 40, 589-595 (1971).

35 Schmid, M., und Ambühl, H.: Bestimmung geringster Mengen von Gesamtphosphor im Wasser von Binnenseen. Schweiz. Z. Hydrol. 27, 183-192 (1965).

36 Sládeček, V.: A Note on the Phytoplankton-Zooplankton Relationship. Ecology 39, 547-549 (1958).

37 Smith, F.A.: Active Phosphate Uptake by Nitella translucens. Biochim. biophys. Acta 126, 94-99 (1966). 
38 Strickland, J.D.H., und Terhune, L.D.B.: The Study of in situ Marine Photosynthesis Using a Large Plastic Bag. Limnol. Oceanogr. 6, 93-96 (1961).

39 Strickler, J.R.: Observations of Swimming Performances of Planctonic Copepods. Limnol. Oceanogr. 22, 165-170 (1977).

40 Uehlinger, U.: Autökologie von Aphanizomenon flos-aquae (in Vorbereitung).

41 Utermöhl, H.: Zur Vervollkommnung der quantitativen Phytoplanktonmethodik. Mitt. int. Verein. Limnol. 2, 1-38 (1958).

42 Vetter, H.: Bemerkungen über das Phytoplankton und seine Beziehungen zur Ernährung des Crustaceenplanktons im Schussenaltwasser und im Bühelweiher. Schr. Ver. Gesch. Bodensees 64 (1937).

43 Vollenweider, R.A.: Scientific Fundamentals of the Eutrophications of Lakes and Flowing Waters, with Particular Reference to Phosphorus and Nitrogen as Factors in Eutrophication. OECD Tech. Rep. DAS/CSI 68.27 (revised 1971).

44 Vollenweider, R.A.: Advances in Defining Critical Loading Levels for Phosphorus in Lake Eutrophication. Mem. Ist. ital. Idrobiol. 33, 53-83 (1976).

45 Volterra, V.: Leçons sur la théorie mathématique de la lutte pour la vie. Gauthier-Villars, Paris 1931.

Adresse der Autoren: Abteilung Limnologie der EAWAG, Überlandstrasse 133, CH-8600 Dübendorf, Schweiz. 